\title{
A 2.4 GHz 1V Low Power LNA in Subthreshold Region
}

\author{
HangjiLiu $^{1,2,3}$, ZhiqunLi $^{1,2}$,MengZhang ${ }^{1}$ \\ ${ }^{1}$ Institute of RF- \& OE-ICs, Southeast University, Nanjing, 210096, China \\ ${ }^{2}$ Key Laboratory of Jiangsu Province Sensor Network Technology, Wuxi, 214135, \\ China \\ ${ }^{3}$ College of Software Engineering, Southeast University, Suzhou 215123, China \\ Corresponding author: zhiqunli@seu.edu.cn
}

\begin{abstract}
Thispaper presents the analysis and design of a 1V lowpower differential low noise amplifier (LNA) in $0.18 \mu \mathrm{m}$ RF CMOS technology for $2.4 \mathrm{GHz}$ WSN applications. The circuit benefits from subthreshold region and $g_{\mathrm{m}}$-boosting techniques. These techniques provide a high gain andreducenoise figure (NF) and power consumption. The performance trade-off of thecircuit is discussed. Post simulation results have been shown and it exhibitsa 16.8dBgainwitha4.9dBNFwhiledissipating $0.9 \mathrm{~mW}$ power from $1 \mathrm{~V} \mathrm{DC}$ supply.
\end{abstract}

Keywords - low noise amplifier (LNA); 1V; low power; subthreshold

\section{INTRODUCTION}

$\mathrm{W}$ IRELESS sensor network (WSN) standards like IEEE 802.15.4 require drastically low power consumption for remote applications with long battery lifetime.In order to achieve long battery life, the power consumption of the WSN receiver must be reduced and maintain an appropriate receiver sensitivity ${ }^{[1-4]}$.

By applying subthreshold region and $g_{\mathrm{m}}$-boosting techniques the supply voltage, current and NFcan be reduced.The aim of this paper is to design a $1 \mathrm{~V}$ low power LNA.

Recently, CGLNA has become more attractive than CSLNA for its wideband input impedance matching. But compare with inductively degenerated common source (CS) LNA topology, thistopology has lower gain, so we choose CG cascade stage as the main topology of the circuit.

Besides, this topology has higher noise figure (NF) under $10 \mathrm{GHz}{ }^{[5]}$, and the NF always has directly impact on the sensitivity of the RF receivers. But, in WSN applications, the nodes are always positioned not long distance, so the receivers do not have to achieve very high sensitivity. The robustness of the circuit, the DC supply voltage, the chip area, and the power consumption are key factors.

This paper is organized as follow: Section II discusses the circuit topology and gives the proposed LNA circuit. The relevant designed considerations of the proposed LNA are described in Section III. Section IV presents the post simulation results of the LNA. Finally, the paper concludes in Section V. 


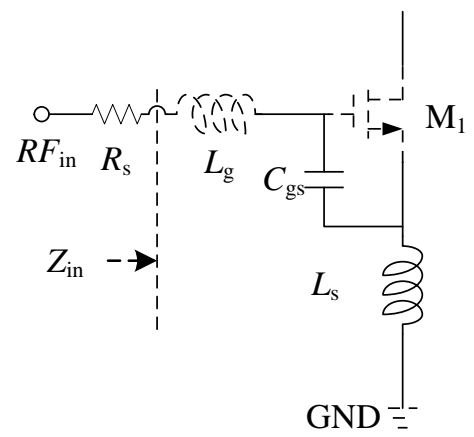

Fig. 1.Schematic of the basic CSLNA

\section{TOPOLOGY DESIGN CONSIDERATION}

The critical benchmarks for characterizing the performance of an LNA are NF, gain, power consumption, reverse isolation, stability, linearity, ease of input matching and matching accuracy usually relative to $50 \Omega^{[6]}$. The currently popular CSLNA topology is shown in Fig. 1 , the input impedance at resonance to $50 \Omega^{[7]}$. Its input impedance can be expressed as

$$
Z_{\mathrm{in}, \mathrm{CSLNA}} \approx s L_{\mathrm{g}}+\omega_{\mathrm{T}} L_{\mathrm{s}}+s L_{\mathrm{s}}+1 / s C_{\mathrm{gs}}
$$

where $\omega_{\mathrm{T}}=g_{\mathrm{m}} / C_{\mathrm{gs}}$ is the unity current gain frequency of the $\mathrm{M}_{1}$.It is hard to do the impedance matching at the inputs due to the interference of the circuit parasitic capacitance, the on-chip inductor modeling deviation, and so on. But an effective series resonance created at the RF operating frequency leads to a noiseless resistive input match, which accounts for the superior noise performance of the CSLNA configuration ${ }^{[6]}$.

As stated in the Section I, it is easier for the CGLNA to doimpedance matching than the CSLNA, which without the influence of parasitic reactance. The input impedance of the CGLNA can be given by ${ }^{[8]}$

$$
Z_{\mathrm{in}, \mathrm{CGLNA}} \approx \frac{R_{\mathrm{D}}}{\left(g_{\mathrm{m}}+g_{\mathrm{mb}}\right) r_{\mathrm{o}}}+\frac{1}{g_{\mathrm{m}}+g_{\mathrm{mb}}}
$$

where $g_{\mathrm{m}}$ is the transconductance of the CG stage transistors, and $R_{\mathrm{D}}$ is the equivalent load connected to the drain of the MOS transistor. The antenna impedance is $50 \Omega$. By adjusting the $g_{\mathrm{m}}$ and the $R_{\mathrm{D}}$, it can obtain fully or partially resistive impedance that is less affected by parasitic reactance. The proposed LNA is shown in Fig.2. 


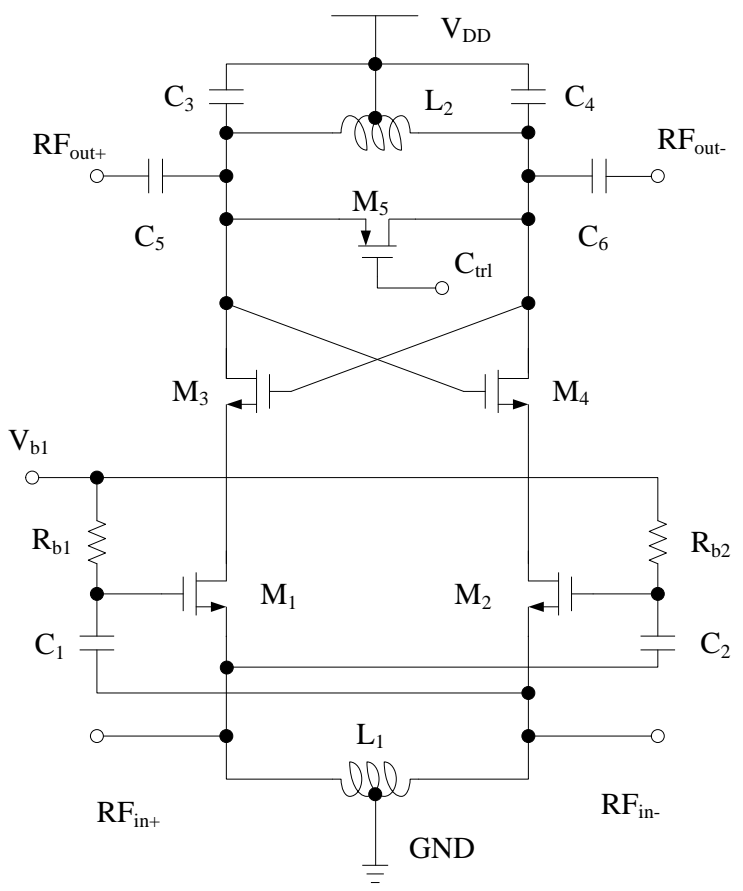

Fig. 2. Schematic of the proposed LNA

\section{Circuit DESIGN}

\section{A. Subthresholdregion}

In reality, for $V_{\mathrm{GS}}$ approaches $V_{\mathrm{T}}$ (threshold voltage), a "weak" inversion layer still exists and some current flows from $\mathrm{D}$ (drain) to $\mathrm{S}$ (source). Even for $V_{\mathrm{GS}}<V_{\mathrm{T}}, I_{\mathrm{D}}$ is finite, but it exhibits an exponential dependence on $V_{\mathrm{GS}}$. Called "subthreshold conduction," this effect can be formulated for $V_{\mathrm{DS}}$ greater than roughly $200 \mathrm{mV}$ as ${ }^{[8-9]}$

$$
I_{\mathrm{D}}=\frac{W}{L} I_{\mathrm{D} 0} \exp \frac{q V_{\mathrm{GS}}}{n k \mathrm{~T}}(3)
$$

wheren $>1$ is nonideality factor. The key factor here is that as $V_{\mathrm{GS}}$ falls below $V_{\mathrm{T}}$, the drain current drops at a finite rate. With typical value of $n$, at room temperature $V_{\mathrm{GS}}$ must decrease by approximately $80 \mathrm{mV}$ for $I_{\mathrm{D}}$ to decrease by one decade, as shown in Fig. $3^{[8]}$. 


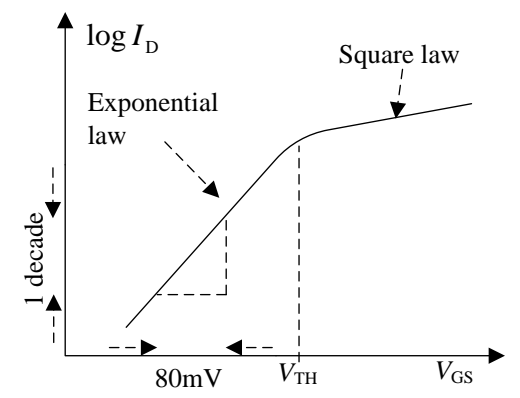

Fig. 3. Plot of drain current of $V_{\mathrm{GS}}$

The exponential relationship between $I_{\mathrm{D}}$ and $V_{\mathrm{GS}}$ inthe subthresholdregion suggests the use of MOS transistors in this regime so as to achieve low drain current. As $I_{\mathrm{D}}$ decreases the power dissipation is lower.

\section{B. Linearity}

In most applications, the LNA does not limit the linearity of the receiver. Owing to the cumulative gain through the RX chain, thelatter stages, e.g., the baseband amplifiers or filters tend to limit the overall $\mathrm{IIP}_{3}$ or $\mathrm{IP}_{1 \mathrm{~dB}}$. We therefore design and optimizeLNAs with little concern for their linearity ${ }^{[10]}$.

But subthreshold region and $g_{\mathrm{m}}$-boosting techniqueshave been applied to reduce the current and noise at the same time, which can also increase the $g_{\mathrm{m}}$ related distortion and introduce extra distortion. Anestimation ofthe $g_{\mathrm{m}}$-boosted LNA IIP 3 using $\mathrm{IIP}_{3}$ andIIP 2 ofboth CGLNA and $g_{\mathrm{m}}$-boosting CGLNA can be given by ${ }^{[11]}$.

$$
\frac{1}{I I P_{3}}=\frac{4}{I I P_{3-C G}{ }^{2}}+\frac{1}{2 I I P_{3-B O O S T}{ }^{2}}+\frac{3}{2} \frac{1}{I I P_{2-C G} \cdot I I P_{2-B O O S T}}
$$

The up-scaling of $g_{\mathrm{m}}$-boosting CGLNA includes a noise-linearity trade-off since a high boosting gain is necessary for lowNF but leads to a degraded linearity performance. Fig.4 depicts the schematic simulated $\mathrm{IIP}_{3}$.

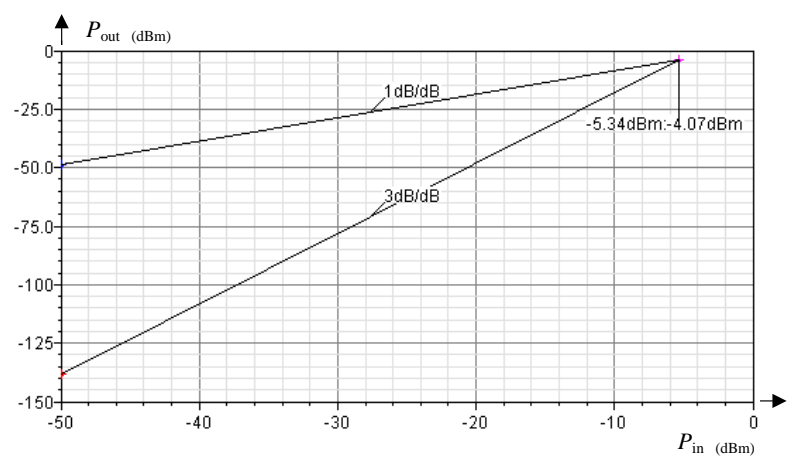

Fig. 4.IIP 3 dBat low gain mode

\section{Stability analysis}

Given the positive feedback in the circuit, the stability must be investigated.The 
small-signal impendence looking into the drain of $\mathrm{M}_{1}$ is $r_{1}$ or $\mathrm{M}_{2}$ is $r_{2}$. As $\mathrm{M}_{1}$ and $\mathrm{M}_{2}$ are identical, so $r_{1}=r_{2}=r$. The equivalent circuit of the positive feedback stage is shown in Fig. 5 . The signals in $\mathrm{M}_{3}$ and $\mathrm{M}_{4}$ are a pair of differential signals, so the connections of $r_{1}$ and $r_{2}$ are equivalent virtual ground. The equivalent impendence $Z_{X}$ from the drain of $M_{3}$ and $M_{4}$ can be written as

$$
\begin{gathered}
V_{\mathrm{X}}=V_{\mathrm{gs} 4}+g_{\mathrm{m} 4} V_{\mathrm{gs} 4} r_{2}+\left(-g_{\mathrm{m} 3} V_{\mathrm{gs} 3} r_{1}\right)-V_{\mathrm{gs} 3} \\
I_{\mathrm{X}}=g_{\mathrm{m} 3} V_{\mathrm{gs} 3}=-g_{\mathrm{m} 4} V_{\mathrm{gs} 4} \\
Z_{\mathrm{X}}=\frac{V_{\mathrm{X}}}{I_{\mathrm{X}}}=\left(1+g_{\mathrm{m}} r\right) \frac{V_{\mathrm{gs} 4}-V_{\mathrm{gs} 3}}{g_{\mathrm{m} 3} V_{\mathrm{gs} 3}}=\left(1+g_{\mathrm{m}} r\right)\left(-\frac{2}{g_{\mathrm{m}}}\right)=-2\left(\frac{1}{g_{\mathrm{m}}}+r\right)
\end{gathered}
$$

where $g_{\mathrm{m} 3}$ is the transconductance of $\mathrm{M}_{3}, g_{\mathrm{m} 4}$ is the transconductance of $\mathrm{M}_{4}$. As $\mathrm{M}_{3}$ and $\mathrm{M}_{4}$ are identical, so $g_{\mathrm{m} 3}=g_{\mathrm{m} 4}=g_{\mathrm{m}}$. But the positive feedback loop may lead the LNA to instability. As $Z_{\mathrm{L}}$ is the equivalent impendence of the $\mathrm{LC}$ parallel resonant network, we should keep $Z_{\mathrm{X}}<-Z_{\mathrm{L}}$ to avoid oscillation. The result can be derived as follow.

$$
\left.\frac{Z_{\mathrm{X}} \cdot Z_{\mathrm{L}}}{Z_{\mathrm{X}}+Z_{\mathrm{L}}}\right|_{Z_{\mathrm{X}}<0, Z_{\mathrm{L}}>0}>0 \Rightarrow Z_{\mathrm{X}}+Z_{\mathrm{L}}<0 \Rightarrow Z_{\mathrm{X}}<-Z_{\mathrm{L}}
$$

During the simulation, we should monitor the stability factor $k$-factor ${ }^{[10]}$, whichindicates unconditional stability when $k_{\mathrm{f}}>1$.Fig. 6 shows the schematic simulated $k_{\mathrm{f}}$.

$$
k \approx \frac{1+\left|S_{11}\right|^{2}-\left|S_{22}\right|^{2}+\left|S_{11} S_{22}-S_{12} S_{21}\right|^{2}}{2\left|S_{12} S_{21}\right|}
$$

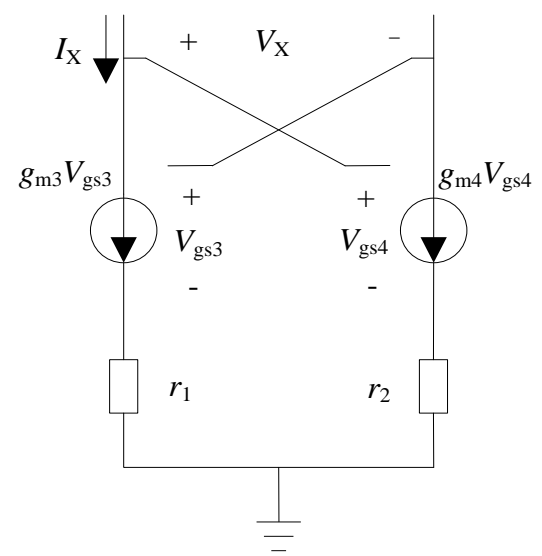

Fig. 5. Equivalent circuit of the positive feedback stage 


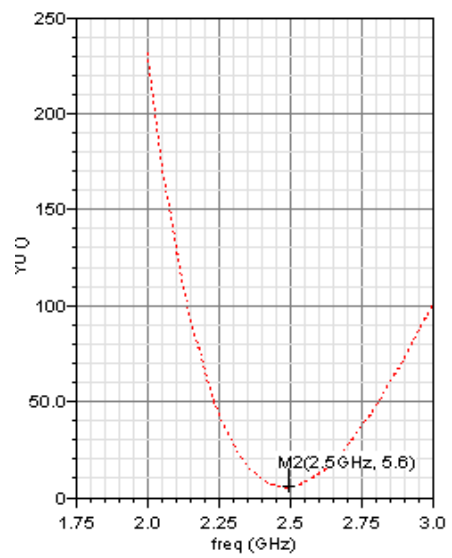

Fig. $6 . k_{\mathrm{f}}$ at high gain mode

\section{POST Simulation RESUltS}

The layout of the LNA is shown in Fig.7. The total chip area is $950 \mu \mathrm{m} \times 560 \mu \mathrm{m}$. The post simulated results of the LNA are shown as follow: S-parameterpost simulatedresults of the LNA are shown in Fig.8 and Fig.9 respectively. Noise figure and NFminare shown in Fig.10 and Fig.11. $\mathrm{IP}_{1 \mathrm{~dB}}$ at high gain mode is shown in Fig.12 and at low gain mode is shown in Fig.13. Table 1 gives a summary of the post simulation results.

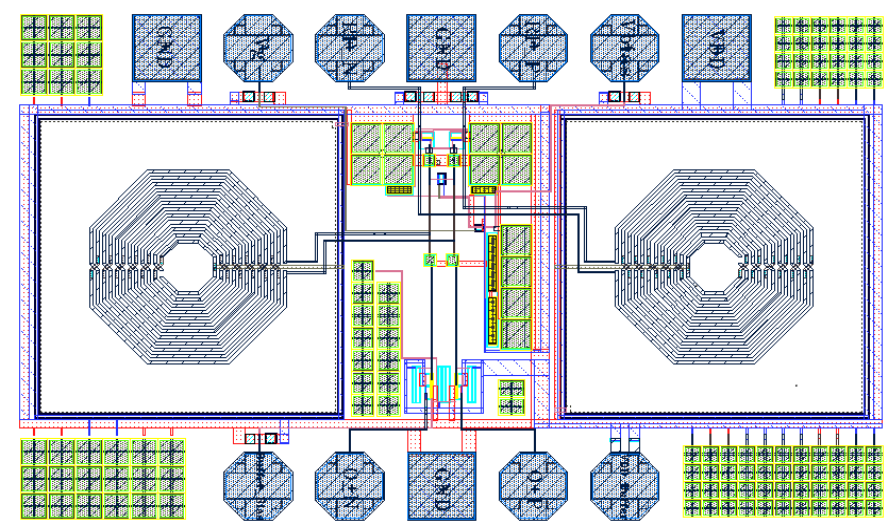

Fig. 7.Layout of the LNA. 

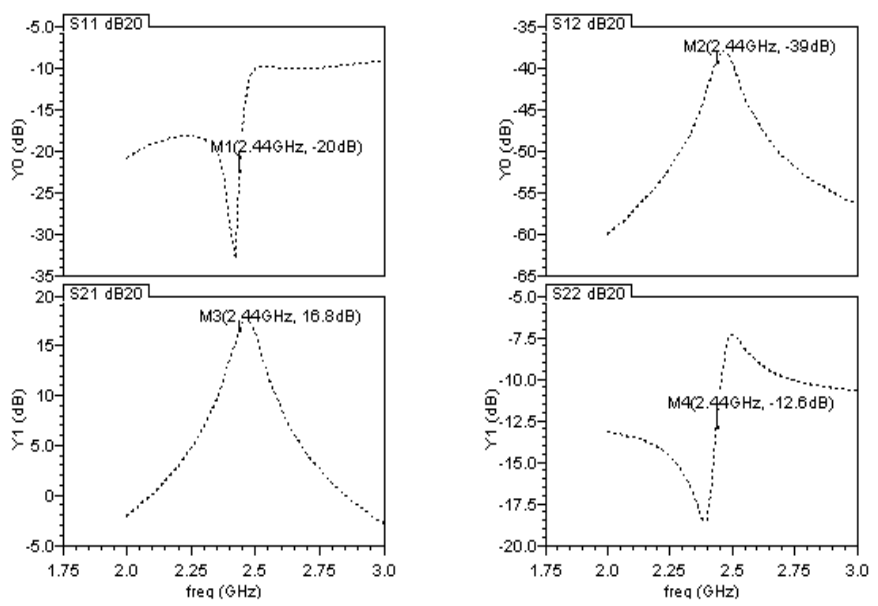

Fig. 8.S-parameter of the LNA at the high gain mode.
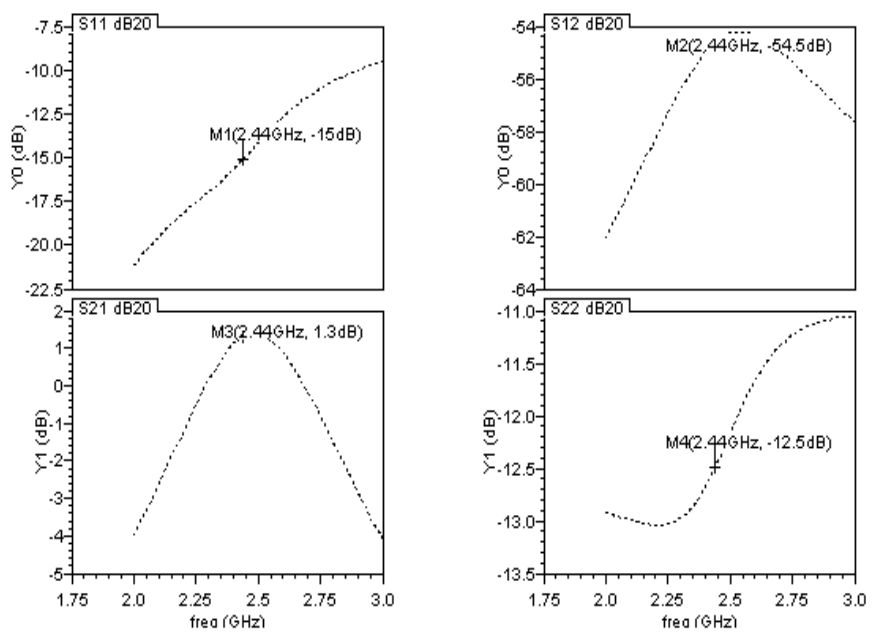

Fig. 9.S-parameter of the LNA at the low gain mode.

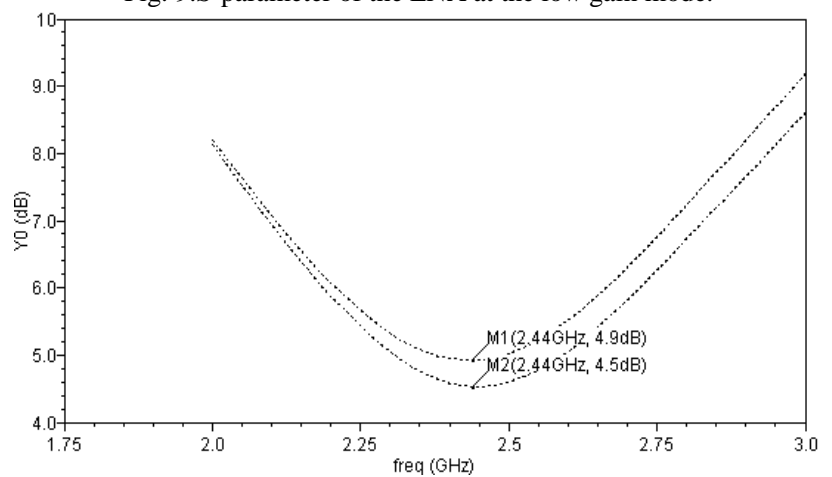

Fig. 10.NF and NFmin at high gain mode. 


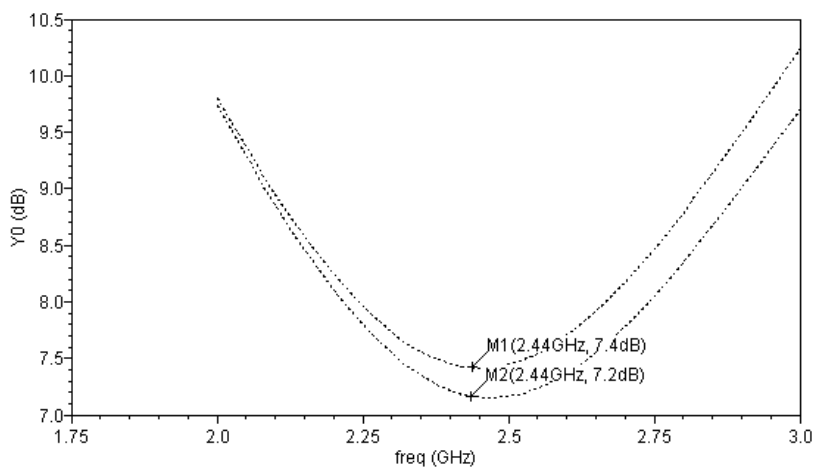

Fig. 11.NF and NFmin at low gain mode.

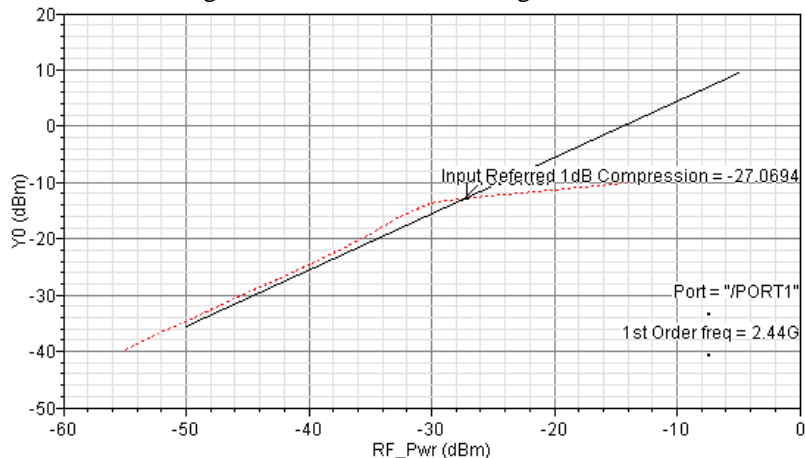

Fig. 12.IP1dB at high gain mode.

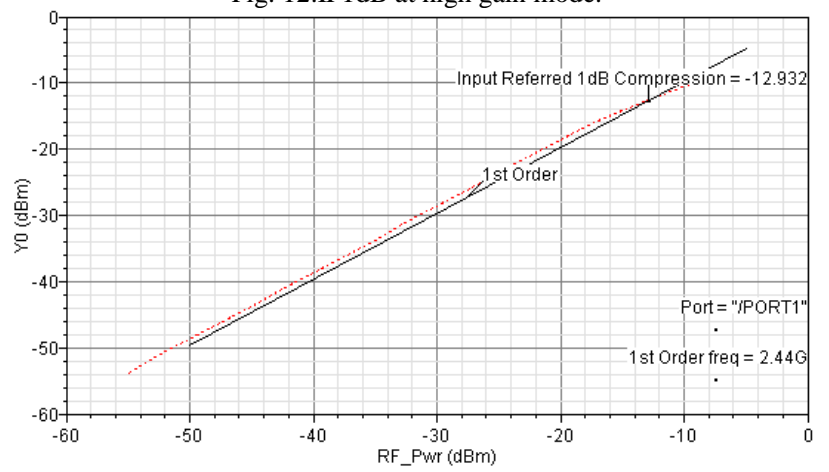

Fig. 13.IP1dB at low gain mode.

Table 1. Simulation Results of LNA

\begin{tabular}{lc}
\hline \multicolumn{1}{c}{ Parameter } & Value \\
\hline Supply voltage & $1 \mathrm{~V}$ \\
DC current & $0.9 \mathrm{~mA}$ \\
Power consumption & $0.9 \mathrm{~mW}$ \\
$\mathrm{~S}_{11}$ & $<-10 \mathrm{~dB}$ \\
$\mathrm{~S}_{21}$ at $2.44 \mathrm{GHz}$ & $1.3-16.8 \mathrm{~dB}$ \\
$\mathrm{~S}_{22}$ & $<-10 \mathrm{~dB}$ \\
$\mathrm{NF}$ at high gain mode & $4.9 \mathrm{~dB}$ \\
$\mathrm{IP}_{1 \mathrm{~dB}}$ at low gain mode & $-12.9 \mathrm{dBm}$ \\
\hline
\end{tabular}




\section{CONCLUSION}

In this paper, a1Vlow power low noise amplifier for wirelesssensor networks applications is presented. The LNA design makes use of subthreshold region and $g_{\mathrm{m}}$-boosting techniques, in order to reducethe LNA's supply voltage and current. Trade-off between NF and linearity has been discussed. The LNA shows good stability as well as good robustness versus technologicalvariations. It achieves a power consumption of $0.9 \mathrm{~mW}$.

\section{ACKNOWLEDGMENT}

The authors would like to acknowledge the "National High Technology Research and Development Program of China" and "Special Fund of Jiangsu Province for the Transformation of Scientific and Technological Achievements" for the financial support.

\section{REFERENCES}

[1] T. Taris, A. Mabrouki, H. Kraimia, Y. Deval, and J.-B. Begueret, "Reconfigurable ultra low power LNA for 2.4 GHz wireless sensor networks," inProc. IEEE ICECS, Dec. 2010, pp. 74-77.

[2] Kluge W, Poegel F, Roller H, et al. A fully integrated 2.4-GHz IEEE 802.15.4-compliant transceiver for ZigBee applications. IEEE J Solid-State Circuits, 2006, 41(12): 2767.

[3] Camus M, Butaye B, Garcia L, et al. A $5.4 \mathrm{~mW} / 0.07 \mathrm{~mm} 2.2 .4 \mathrm{GHz}$ front-end receiver in $90 \mathrm{~nm}$ CMOS for IEEE 802.15.4. WPAN standard. IEEE J Solid-State Circuits, 2008, 43(6): 1372.

[4] Zhang Hao, Li Zhiqun, Zhang Meng, et al. A $2.4 \mathrm{GHz}$ low-IF RF frontend for wireless sensor networks. Microwave and Millimeter. Wave Technology (ICMMT), 2010: 225.

[5] Nguyen T K, Kim C H, Ihm G J, et al. CMOS low-noise amplifier design optimization techniques. IEEE Trans Microw Theory Tech, 2004, 52(5): 433.

[6] W. Zhuo, X. Li, S. Shekhar, S. H. K. Embabi, J. Pineda de Gyvez, D. J. Allstot, and E. Sanchez-Sinencio. A capacitor cross-coupled common-gate low-noise amplifier. IEEE TRANSACTIONS ON CIRCUITS AND SYSTEMS-II: EXPRESS BRIEFS, VOL. 52, NO. 12, DECEMBER 2005.

[7] D. K. Shaeffer and T. H. Lee, “A 1.5-V, 1.5-GHz CMOS low-noise amplifier,"IEEE J. Solid-State Circuits, vol. 32, no. 5, pp. 745-759, May 1977.

[8] Razavi B. Design of analog CMOS integrated circuits. Xi'an: Xi'an Jiaotong University Press,2003.

[9] P. R. Gray, P. J. Hurst, S. H. Lewis, and R. G. Meyer, Analysis and Design of Analog Integrated Circuits, 4th ed. New York: Wiley, 2001, ch. 1

[10] Razavi B. RF Microelectronics 2nd. Bei Jing: PUBLISHING HOUSE OF ELECTRONICS INDUSSTRY,2012.

[11] I. R. Chamas and S. Raman, "Analysis, design, and X-band implementation of a self-biased active feedback $g_{\mathrm{m}}$ -boosted common-gate CMOS LNA,"IEEE Trans. Microw. Theory Tech., vol. 57, no. 3, pp.542-551, Mar. 2009. 